ARTICLE

Received 17 Jul 2014 | Accepted 7 Oct 2014 | Published 24 Nov $2014 \quad$ DOl: 10.1038/ncomms6494

\title{
Retinoid $X$ receptor $\alpha$ attenuates host antiviral response by suppressing type I interferon
}

\author{
Feng Ma ${ }^{1, \star}$, Su-Yang Liu ${ }^{1, \star}$, Bahram Razani ${ }^{1}$, Neda Arora ${ }^{1}$, Bing $\mathrm{Li}^{2}$, Hiroyuki Kagechika ${ }^{3}$, Peter Tontonoz ${ }^{4,5}$, \\ Vanessa Núñez ${ }^{6}$, Mercedes Ricote $^{6} \&$ Genhong Cheng ${ }^{1}$
}

The retinoid $X$ receptor $\alpha(R X R \alpha)$, a key nuclear receptor in metabolic processes, is downregulated during host antiviral response. However, the roles of RXR $\alpha$ in host antiviral response are unknown. Here we show that RXR $\alpha$ overexpression or ligand activation increases host susceptibility to viral infections in vitro and in vivo, while Rxra-/ - or antagonist treatment reduces infection by the same viruses. Consistent with these functional studies, ligand activation of RXR inhibits the expression of antiviral genes including type I interferon (IFN) and Rxra-/ - macrophages produce more IFN $\beta$ than WT macrophages in response to polyl:C stimulation. Further results indicate that ligand activation of RXR suppresses the nuclear translocation of $\beta$-catenin, a co-activator of IFN $\beta$ enhanceosome. Thus, our studies have uncovered a novel RXR-dependent innate immune regulatory pathway, suggesting that the downregulation of RXR expression or RXR antagonist treatment benefits host antiviral response, whereas RXR agonist treatment may increase the risk of viral infections.

\footnotetext{
${ }^{1}$ Department of Microbiology, Immunology, and Molecular Genetics, University of California, Los Angeles, California 90095, USA. ${ }^{2}$ Department of Biologica Chemistry, University of California, Los Angeles, California 90095, USA. ${ }^{3}$ Institute of Biomaterials and Bioengineering, Tokyo Medical and Dental University, Tokyo 113-8510, Japan. ${ }^{4}$ Howard Hughes Medical Institute, Chevy Chase, Maryland 20815, USA. ${ }^{5}$ Department of Pathology and Laboratory Medicine, University of California, Los Angeles, California 90095, USA. ${ }^{6}$ Cardiovascular Development and Repair Department, Centro Nacional de Investigaciones Cardiovasculares (CNIC), Melchor Fernandez Almagro 3, 28029 Madrid, Spain. ${ }^{*}$ These authors contributed equally to this work. Correspondence and requests for materials should be addressed to G.C. (email: gcheng@mednet.ucla.edu).
} 
$\mathrm{R}$ etinoid X receptors (RXRs) have been implicated in the control of a variety of physiological processes including cell differentiation, lipid and glucose metabolism and immune responses ${ }^{1-4}$. They occupy a central position in the nuclear hormone receptor (NR) superfamily, because they not only act as homodimers but are also essential heterodimeric partners of many other NR family members, such as retinoic acid receptor (RAR), vitamin $\mathrm{D}$ receptor (VDR), thyroid receptor, liver $\mathrm{X}$ receptor (LXR), peroxisome proliferator-activated receptor (PPAR), pregnane $\mathrm{X}$ receptor and farnesoid $\mathrm{X}$ receptor ${ }^{5,6}$. Heterodimerization with partners raises the possibility of pleiotropic RXR signalling, different RXR isotypes and their unique expression increase the complexity of the RXR-controlled transcriptional programs. Mammalian tissue express three RXR isotypes, $\operatorname{RXR} \alpha, \operatorname{RXR} \beta$ and $\operatorname{RXR} \gamma$, which have specific tissue distributions and expression patterns during development ${ }^{6-11}$. $\mathrm{RXR} \alpha$ is the dominant and the most functionally important RXR isotype in myeloid cells, especially macrophages ${ }^{12,13}$. All RXR isotypes can be activated by natural and synthetic ligands. The vitamin A derivative, retionid 9-cis-retinoic acid (9cRA), is identified as a high-affinity ligand for $\mathrm{RXR}^{14,15}$. Endogenous fatty acids such as docosahexaenoic acid, oleic acid and phytanic acid, also activate $\mathrm{RXR}^{7}$. Synthetic RXR-specific ligands, called rexinoids, are exemplified by LG100268 (LG268), AGN194204, as well as Targretin (bexarotene), which is a pan-RXR agonist used clinically for the treatment of T-cell lymphoma ${ }^{15-17}$.

RXRs regulate the integration of macrophage immune functions and lipid metabolism by controlling apoptotic cell uptake, $\beta$-amyloid clearance, inflammation, pathogen killing, cholesterol transport and lipid handling. Alterations in these RXR-mediated processes cause diseases such as atherosclerosis, neurodegeneration, autoimmunity and disorders of the immune response ${ }^{4,11}$. We previously showed that $\operatorname{RXR} \alpha$ expression was significantly suppressed during host defence against viral infections. Downregulation of $\operatorname{RXR} \alpha$ decreases the ability of RXR-dependent hepatic detoxification and thus enhances bile acid- and aspirin-induced hepatotoxicity, which provides evidence that alteration of RXR expression plays a role in the pathogenesis of metabolic disease such as Reye's syndrome ${ }^{18}$.

The crosstalk between RXR and inflammatory responses in macrophages has been extensively studied. Ligand activation of LXR-RXR heterodimer inhibits Toll-like receptor-triggered iNOS, interleukin (IL)-1 $\beta$, COX2 and IL-6 production ${ }^{19}$. In lipopolysaccharide-stimulated macrophages, ligand activation of RAR-RXR heterodimer reduces synthesis of inflammatory cytokines, IL-12 and tumour necrosis factor- $\alpha$, and increases the expression of anti-inflammatory cytokine, IL-10 (refs 20,21). $\mathrm{RXR} \alpha$ also controls innate inflammatory responses through the upregulation of the chemokine CCL6 and CCL9 (ref. 13). Defect of LXR-RXR signalling increases host susceptibility to Listeria monocytogenes infection ${ }^{22}$. Activation of VDR-RXR leads to the induction of cathelicidin and defensin beta 4 genes, which are essential in combating Mycobaterium tuberculosis ${ }^{23}$. While RXR and inflammatory responses are evidently connected, our previous study implicated a relationship between the host antiviral response and $\mathrm{RXR} \alpha^{18}$. It is still unclear whether downregulation of $\operatorname{RXR} \alpha$ during viral infection plays a role in the overall host antiviral response. Meanwhile, considering that RXRs regulate the expression of inflammatory cytokines, antiinflammatory cytokines, and chemokines, it is an interesting issue whether RXRs also modulate the production of type I interferon (IFN) in macrophages.

The innate cellular antiviral response involves numerous signalling pathways that generally lead to the induction of type I IFN. Briefly, viral nucleic acids are recognized by pathogen recognition receptors, such as Toll-like receptor, RIG-I, MDA5,
IFI16 and cGAS ${ }^{24,25}$. These sensors trigger pathways that lead to the activation of tank-binding kinase 1 (TBK1), phosphorylation and nuclear translocation of IFN regulatory factor 3 (IRF3), which subsequently drives type I IFN transcription ${ }^{24-26}$. Type I IFN induces multiple antiviral genes expression through the JakStat $1 / 2$ pathway. Some genes, like ISG15, GBP1 and OAS2, directly inhibit viral lifecycle processes ${ }^{27}$. Others like IRF1, IRF7 and the viral nucleic acid sensors (RIG-I and MDA5) further amplify the IFN response ${ }^{24,25}$. Recent studies have indicated that $\beta$-catenin acts as a co-activator in the IFN $\beta$ enhanceosome and synergistically activate IFN $\beta$ transcription during host antiviral response ${ }^{28,29}$. Sendai virus, vesicular stomatitis virus (VSV), and herpes simplex virus-1 (HSV-1) infections trigger host $\beta$-catenin nuclear translocation by activating $\beta$-catenin phosphorylation and deacetylation ${ }^{29-31}$. The relationship of these pathways critical in host antiviral response and RXR remains unknown.

In this study, we demonstrate that $\operatorname{RXR} \alpha$ attenuates host antiviral response by inhibiting type I IFN production and its downstream IFN-stimulated genes (ISGs) expression. We also provide evidence that the suppression of $\beta$-catenin nuclear translocation accounts for the downregulation of antiviral genes by RXR signalling. These results illustrate a molecular crosstalk between host antiviral response and metabolic nuclear receptors, suggesting that the downregulation of $\operatorname{RXR} \alpha$ expression in response to viral infection is beneficial for optimal type I IFN production and host antiviral immunity.

\section{Results}

Antiviral immune response suppresses $R X R \alpha$ expression. Our previous gene expression profile has shown that VSV infection downregulates $\operatorname{RXR} \alpha$ expression in liver ${ }^{18}$. Further studies have indicated that TLR3- or TLR4-activated macrophages suppress RXR $\alpha$ expression in an IRF3-depedent but IFN-independent manner ${ }^{18}$. To verify the previous conclusions, we tried different viral infection models in bone marrow-derived macrophages (BMMs). BMMs were infected with RNA virus, VSV, and DNA viruses, HSV-1 and murine gamma herpes virus (MHV68). Quantitative PCR (qPCR) results indicated that infections with both RNA and DNA viruses led to significant downregulation of RXR $\alpha$ expression (Fig. 1a). To exclude certain viral components, such as viral inhibitory noncoding RNAs and proteins, suppressing host $\mathrm{RXR} \alpha$ expression, the cells were transfected with polyI:C and polydA:dT, which mimic RNA and DNA viral infections, respectively. We observed that transfection with polyI:C and polydA:dT downregulated $\mathrm{RXR} \alpha \mathrm{RNA}$ and protein expression in BMMs (Fig. 1b,c). These experiments suggest that viral infection suppresses $\mathrm{RXR} \alpha$ expression in BMMs and this suppression is due to the host antiviral response.

Alteration of $\mathrm{RXR} \alpha$ expression affects VSV infection. Alteration of $\operatorname{RXR} \alpha$ expression causes numerous metabolic and immune diseases ${ }^{11,18}$. To test whether alteration of $\operatorname{RXR} \alpha$ expression affects host antiviral response, we performed in vitro infections of hRXR $\alpha$-overexpressing and Rxra-/- cell lines with VSV and quantified the viral titres in the supernatant of the infected cells by plaque assay. First, we stably overexpressed human $\operatorname{RXR} \alpha$ and two $\operatorname{RXR} \alpha$ mutants in a macrophage cell line, RAW264.7 cells (Supplementary Fig. 1a,b). Overexpression of wild-type (WT) human $\operatorname{RXR} \alpha$ made the cells much more susceptible to VSV infection (Fig. 1d). The lysine-108 residue within the $\operatorname{RXR} \alpha$ AF1 domain mediates $\mathrm{RXR} \alpha$ sumoylation during ligand activation, which negatively regulates the transcriptional activity of $\operatorname{RXR} \alpha$ and the activity of its heterodimeric complex with RAR $\alpha$ and PPAR $\gamma^{32}$. Prevention of $\mathrm{RXR} \alpha$ sumoylation by Lys-to-Arg mutation led to modest 
a

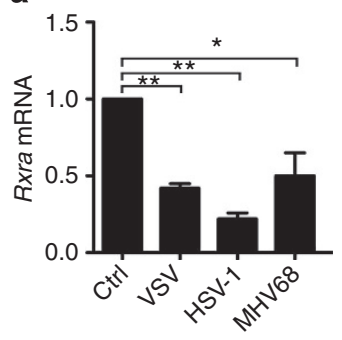

b

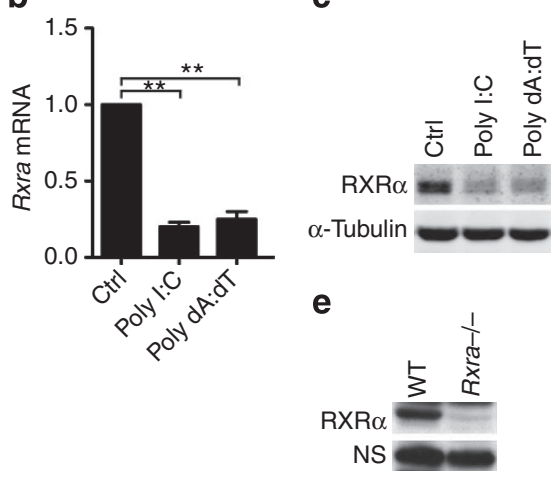

d

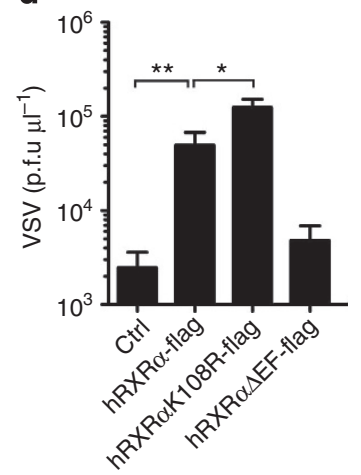

f

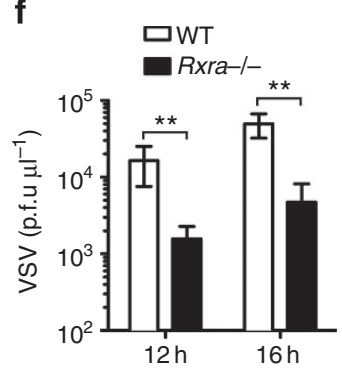

g

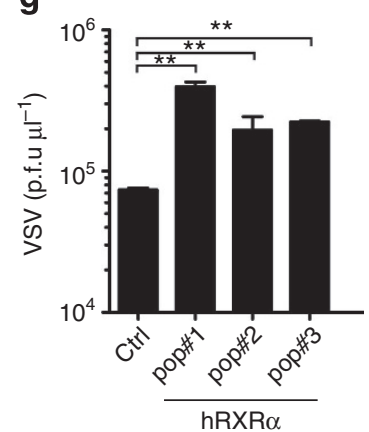

Figure 1 | Host susceptibility to VSV infection after altering RXR $\alpha$ expression. (a) qPCR detection of Rxra mRNA in BMMs infected by VSV for $4 \mathrm{~h}$, HSV1 or MHV68 for $8 \mathrm{~h}(\mathrm{MOI}=1)$. (b) qPCR detection of Rxra mRNA in BMMs transfected with $1 \mu \mathrm{g} \mathrm{ml}{ }^{-1}$ polyl:C or polydA:dT for $4 \mathrm{~h}$. (c) Immunoblot of $\mathrm{RXR} \alpha$ protein in BMMs transfected with $1 \mu \mathrm{g} \mathrm{ml} \mathrm{m}^{-1}$ polyl:C or polydA:dT for $8 \mathrm{~h}$. (d) RAW264.7 cells stably transfected with pBABE-empty vector (Ctrl), pBABE-hRXR $\alpha$-flag vector (hRXR $\alpha$-flag), RXR $\alpha$ sumoylation site mutant (hRXR $\alpha$ K108R-flag) and RXR $\alpha$ LBD-AF2-domain-deficient mutant (hRXR $\alpha \Delta$ EFflag). These cells were infected by VSV $(\mathrm{MOI}=0.01)$. Supernatants were collected at 14 h.p.i and viral titre was quantified by plaque assay. $(\mathbf{e})$ Immunoblot of RXR $\alpha$ protein in WT and Rxra - / - F9 cells. (f) WT or Rxra - / - cells were infected by VSV $(\mathrm{MOI}=0.01)$ and the viral titre of supernatant was quantified at indicated time point by plaque assay. (g) Rxra - / - F9 cells were reconstituted with pBABE-hRXR $\alpha$ (hRXR $\alpha$ ) or pBABE-empty vector (Ctrl) by retroviral infection. Three independent hRXR $\alpha$-overexpressing populations (pop\#1-3) were made, these cells were infected by VSV (MOI $=0.01$ ). Supernatants were collected at 12 h.p.i and the viral titre was quantified for by plaque assay. Data of $\mathbf{a}, \mathbf{b}, \mathbf{d}, \mathbf{f}, \mathbf{g}$ are shown as mean \pm s.d. ( $n=3$ ) of one representative experiment, similar results were obtained in three independent experiments. ${ }^{\star} P<0.05$ and ${ }^{\star \star} P<0.01$ (Student's $t$-test). Data of $\mathbf{c}$ and $\mathbf{e}$ are representative of three independent experiments. See also Supplementary Fig. 7 of uncropped blot of $\mathbf{c}$ and $\mathbf{e}$

increase of VSV infection (Fig. 1d). However, deletion of the ligand binding/dimerization domain and helix 12 (AF2) of RXR $\alpha$ abolished the ability of RXR to increase host susceptibility to VSV infection, suggesting that the ligand-independent $R X R \alpha$ mutant is not sufficient to affect the host antiviral response (Fig. 1d). Next, we took advantage of WT and Rxra - / - F9 embryocarcinoma cells, which have been extensively used to study the function of RXRs in retinoid signalling ${ }^{33,34}$ (Fig. 1e). When these cells were infected with VSV, the viral titre was lower in the supernatant of Rxra - / - F9 cells compared with that of the WT cells (Fig. 1f). To confirm that less VSV infection was specifically due to deficiency of $\mathrm{RXR} \alpha$, we rescued the Rxra-/- F9 cells with $\operatorname{hRXR} \alpha$ (Supplementary Fig. 1c) and found that $\operatorname{hRXR} \alpha$ reconstitution led to an increase in the VSV titre after infection compared with control (Fig. 1g). Together, the overexpression of $\operatorname{RXR} \alpha$ increases host susceptibility to VSV infection and knockout of RXR $\alpha$ decreases host susceptibility to VSV infection.

Ligand activation of RXR promotes VSV infection. Ligand binding to RXR is required for its downstream signalling and full activation. 9cRA is a high-affinity ligand for RXR and RAR. HX531 is a synthetic RXR antagonist, which could inhibit the activation of both RXR homodimer and RXR-RAR heterodimer $^{35}$. To investigate whether ligand activation of RXR affects host susceptibility to viral infection, RAW264.7 cells were pretreated with 9cRA and HX531 overnight, and subsequently infected with green fluorescent protein (GFP)-expressing VSV (VSV-GFP), which allows the visualization of viral infection by fluorescence microscopy. Apparently, there was more VSV-GFP in the 9cRA-treated cells and less VSV-GFP in the HX531-treated cells than the control dimethylsulphoxide (DMSO)-treated cells (Supplementary Fig. 2a). The plaque assay data also indicated that 9cRA treatment promotes VSV infection in a dose-dependent manner and HX531 treatment enhanced host cells against VSV infection (Fig. 2a and supplementary Fig. 2b). We tried other two synthetic RXR-specific agonists, LG268 and AGN194204. Significant higher VSV titre was detected in the supernatant of LG268- and AGN194204-treated cells, which indicated that both of them increased host susceptibility to VSV infection as 9cRA (Fig. 2b). Combination of AGN194204 treatment and hRXR $\alpha$ overexpression in RAW264.7 further increased VSV infection (Supplementary Fig. 2c). In F9 cells, treatment with AGN194204 promoted VSV infection in WT cells, but not in Rxra - / - cells (Fig. 2c). However, after we reconstituted hRXR $\alpha$ into Rxra - / F9 cells, hRXR $\alpha$-reconstituted cells were infected more VSV than control cells, and AGN194204 treatment further increased host susceptibility to VSV infection (Fig. 2d).

Besides comparing the VSV titre in the supernatant from DMSO- and 9cRA-treated RAW264.7 cells, we also compared 
a

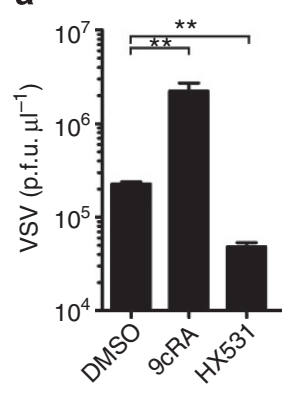

b

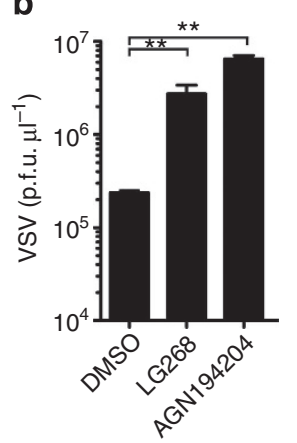

C

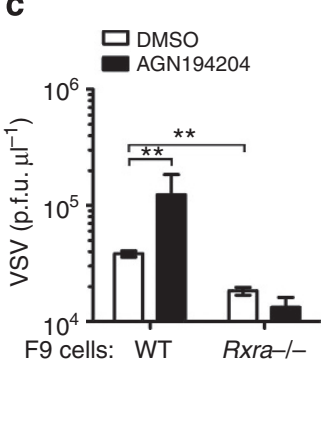

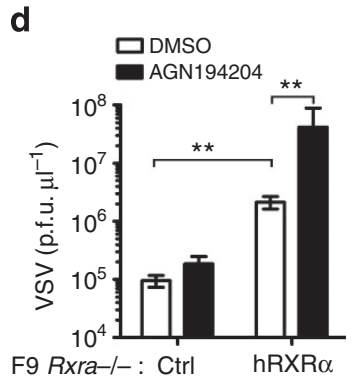

e

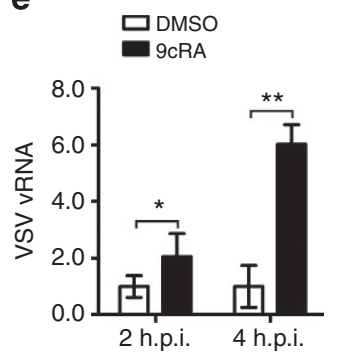

f

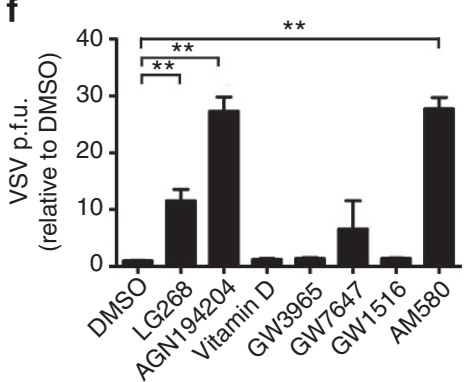

Figure 2 | Host susceptibility to VSV infection after ligand activation of RXR. (a) RAW264.7 cells were pretreated with DMSO, 9cRA (100 nM) and HX531 $(1 \mu \mathrm{M})$ for $16 \mathrm{~h}$ and infected by VSV-GFP $(\mathrm{MOI}=0.01)$. The supernatant was collected at $14 \mathrm{~h} . \mathrm{p} . \mathrm{i}$ and the viral titre was quantified by plaque assay. (b) RAW264.7 cells were pretreated with DMSO or RXR-specific synthetic ligands, LG268 (100 nM) and AGN194204 (100 nM) for 16 h. The cells were subsequently infected by VSV $(\mathrm{MOI}=0.01)$ and the supernatant was collected at $14 \mathrm{~h}$. ..i. The viral titre was quantified by plaque assay. (c) WT and Rxra - / - F9 cells were pretreated with DMSO or AGN194204 $(100 \mathrm{nM})$ for $16 \mathrm{~h}$ and subsequently infected with VSV (MOI $=0.01)$. The viral titre of supernatants was quantified by plaque assay at 12 h.p.i. (d) Rxra - / - F9 cells stably transfected with pBABE-empty vector (Ctrl) or pBABE-hRXR $\alpha$ $(\mathrm{hRXR} \alpha)$ were treated with DMSO or AGN194204 $(100 \mathrm{nM})$ and infected with VSV $(\mathrm{MOI}=0.01)$. The viral titre of supernatants was quantified by plaque assay at 12 h.p.i. (e) RAW264.7 cells were pretreated with DMSO or 9cRA (100 nM) for $16 \mathrm{~h}$ and infected by VSV (MOI $=0.01)$, relative genomic VSV RNA was quantified at 2 and 4 h.p.i. (f) RAW264.7 cells were pretreated with DMSO, RXR agonist LG268 (100 nM), AGN194204 (100 nM), VDR agonist Vitamin D (100 nM), LXR agonist GW3965 (100 nM), PPAR $\alpha$ agonist GW7647 (100 nM), PPAR $\delta$ agonist GW1516 (100 nM) or RAR agonist AM580 $(100 \mathrm{nM})$ for $16 \mathrm{~h}$ and subsequently infected by VSV $(\mathrm{MOI}=0.01)$. The supernatants were collected at $14 \mathrm{~h} . \mathrm{p} . \mathrm{i}$ and viral titre was detected by plaque assay. Data are shown as mean \pm s.d. $(n=3)$ of one representative experiment, similar results were obtained in three independent experiments. ${ }^{\star} P<0.05$ and ${ }^{\star \star} P<0.01$ (Student's $t$-test).

VSV genomic RNA and VSV-G protein level. More VSV genomic RNA was detected in 9cRA-treated cells when compared with DMSO-treated cells (Fig. 2e). Consistently, more VSV-G protein was observed in both control cells and $\mathrm{hRXR} \alpha$-overexpressing cells after 9cRA treatment (Supplementary Fig. 2d). To determine whether RXR agonist and antagonist affect VSV in the first round infection or subsequent infection processes, we used a VSV pseudovirus that has the receptor-binding G protein (VSV-G) replaced by the luciferase reporter (VSV $\Delta \mathrm{G}-\mathrm{Luc}$ ) enveloped inside VSV-G, called VSV $\Delta G-L u c / G^{36}$. This pseudovirus can undergo VSV-G-mediated entry but cannot produce its own VSV-G envelope, and hence is only capable of a single-round infection. Significant higher luciferase activity was detected in the cells treated with 9cRA and decreased luciferase activity in the cells treated with HX531 (Supplementary Fig. 2e). These results indicate that the RXR agonist and antagonist affect VSV singleround infection.

Given that RXR $\alpha$ forms a homodimer or heterodimer to regulate its target genes ${ }^{6,11}$, we screened agonists for different RXR potential partners including VDR, LXR, PPAR $\alpha$, PPAR $\delta$ and RAR. We found that only RAR ligands could promote VSV infection as RXR-specific ligands, which implies that RXR homodimer or RXR/RAR heterodimer mediates the increased host susceptibility to VSV infection (Fig. 2f).

Taken together, the overexpression of $\operatorname{RXR} \alpha$ or ligand activation of RXR increases host susceptibility to VSV infection, while the knockout of RXR $\alpha$ or antagonist inhibition of RXR decreases host susceptibility to VSV infection.

Ligand activation of RXR $\alpha$ promotes DNA virus infection. Host RXR $\alpha$ expression is downregulated after infection by both RNA and DNA virus. To test if RXR $\alpha$ modulates host response against DNA virus infection as VSV infection, $\mathrm{hRXR} \alpha$-expressing RAW264.7 cells were treated with AGN194204, and infected by DNA viruses, MHV68 and HSV-1. Since MHV68 expressed luciferase, we compared MHV68 infection in DMSO-treated control cells and AGN194204-treated cells by luciferase activity. AGN194204 treatment dramatically increased MHV68 luciferase activity, which indicated the ligand activation of RXR-promoted MHV68 infection (Fig. 3a). AGN194204 treatment also increased HSV-1 viral infection significantly as measured by plaque assay (Fig. 3b). The overexpression of hRXR $\alpha$ alone slightly increased MHV68 luciferase expression and HSV-1 viral load, whereas treatment with 9cRA significantly increased host cell susceptibility to these viruses (Fig. 3c,d). Consistently, the inhibition of RXR activity by HX531 suppressed MHV68 and HSV-1 infections (Fig. 3c,d). These results indicate that ligand activation of RXR also increases host susceptibility to DNA viruses.

Retinoids have been commonly used as drug treatments for skin diseases and various cancers ${ }^{3,11}$. 13-cis-retinoic acid (13cRA), also called Isoretinoin, is a ligand for RAR-RXR 
a

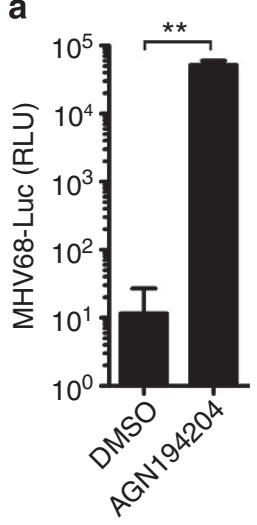

b

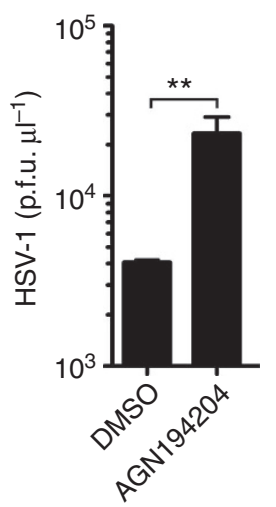

C

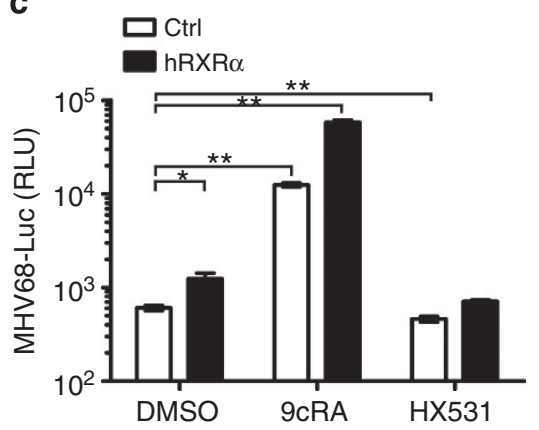

d

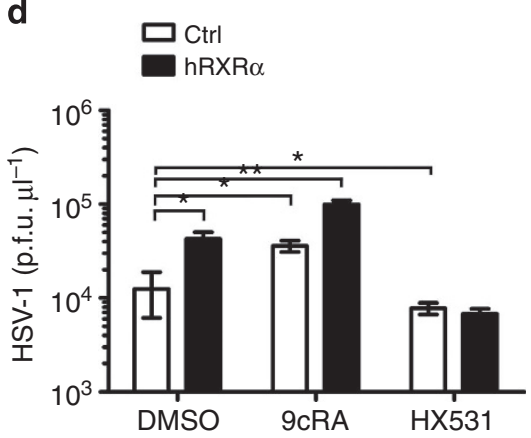

e

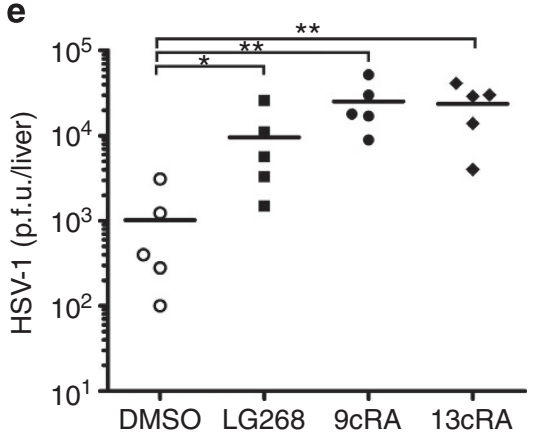

Figure 3 | Host susceptibility to DNA viral infection after pretreating with RXR agonists and antagonist. (a) hRXR $\alpha$-overexpressing RAW264.7 cells were treated with DMSO or AGN19420 (100 nM) for $16 \mathrm{~h}$ and subsequently infected by luciferase-expressing MHV68 (MHV68-luc; MOI=0.5). Cells were lysed in passive lysis buffer at 24 h.p.i and the luciferase activity was quantified by luminescence assay. (b) hRXR $\alpha$-overexpressing RAW264.7 cells pretreated with DMSO or AGN19424 (100 nM) and then these cells were infected by HSV-1 $(\mathrm{MOI}=0.25)$. Supernatants were collected at 24 h.p.i and the viral titre was quantified by plaque assay. (c) RAW264.7 cells stably expressing pBABE control vector (Ctrl) or pBABE-hRXR $\alpha$ vector (hRXR $\alpha$ ) were pretreated with DMSO, 9cRA $(100 \mathrm{nM})$ or HX531 $(1 \mu \mathrm{M})$ for $16 \mathrm{~h}$, and then infected by MHV68-luc $(\mathrm{MOI}=0.5)$. Cells were lysed in passive lysis buffer at 24 h.p.i and the luciferase activity was quantified by luminescence assay. (d) RAW264.7 cells stably expressing Ctrl or hRXR $\alpha$ vector were pretreated with DMSO, 9cRA $(100 \mathrm{nM})$ or HX531 $(1 \mu \mathrm{M})$ for $16 \mathrm{~h}$, and then infected by HSV-1 (MOI $=0.25)$. Supernatants were collected at $24 \mathrm{~h} . p . i$ and the viral titre was quantified by plaque assay. (e) Mice were i.p. injected with DMSO, LG268, 9cRA or 13cisRA ( $25 \mu \mathrm{g}$ per mice) daily for 4 days and infected with $10^{7}$ p.f.u. of HSV-1 on day 5. Mice were killed at 5 d.p.i and the livers were harvested, homogenized in media and quantified for viral titres by plaque assay. Data are representative of two independent experiments, ${ }^{\star} P<0.05$ and ${ }^{\star \star} P<0.01$ (Mann-Whitney test). Data of a-d are shown as mean \pm s.d. ( $n=3$ ) of one representative experiment, similar results were obtained in three independent experiments. ${ }^{\star} P<0.05$ and ${ }^{\star \star} P<0.01$ (Student's $t$-test).

heterodimer and widely used in clinical settings ${ }^{37-39}$. Like 9cRA, 13cRA also promoted VSV infection in RAW264.7 cells (Supplementary Fig. 3). To test whether retinoids can affect HSV-1 infection in vivo, DMSO or different retinoids were administered by intraperitoneal (i.p.) injection to age and sex-matched mice daily for 4 days. The mice were infected with HSV-1 and the livers were harvested on 5 days post infection. Treatment of RXR ligands, LG268, 9cRA and 13cRA, significantly increased HSV-1 titre in mice livers (Fig. 3e). These data are not only supported our in vitro results, but also consistent with the previously reported cases showing that 13cRA may be associated to herpes viral infection ${ }^{40,41}$.

Together, ligand activation of RXR attenuates host antiviral response against both RNA virus and DNA virus.

Activation of $\operatorname{RXR} \alpha$ suppressed multiple antiviral genes. Since $\operatorname{RXR} \alpha$ activation increased host susceptibility to both RNA and DNA viruses, we hypothesized that RXR $\alpha$ caused cellular attenuation of host antiviral response rather than direct enhancement of viral fitness. To test this hypothesis, we performed a microarray to compare the gene expression profiles between DMSO- and 9cRA-treated macrophages after
polyI:C transfection. PolyI:C transfection induced $14.88 \%$ genes and suppressed $9.42 \%$ genes over twofold. These polyI:Cinducible and polyI:C-suppressed genes were considered as polyI:C-responsive genes (Fig. 4a). The polyI:C-responseive genes were further grouped into 9cRA-inducible polyI:Cresponsive genes (Fig. 4a and Supplementary Dataset 1) and 9cRA-suppressed polyI:C-responsive genes (Fig. 4a and Supplementary Dataset 2). Interestingly, among the 9cRA-suppressed polyI:C-responsive genes, there were multiple type I IFN genes (Ifnb1, Ifna2, Ifna4, Ifna5 and Ifna9) as well as Isg15 (Fig. 4b). Given that numerous antiviral genes such as type I IFN and its downstream ISGs can be induced during viral infection and play critical roles in host immune responses against viral infection $^{25,28}$, RXR-mediated suppression on these antiviral genes may be responsible for the increased host susceptibility to viral infection.

To verify the microarray data and support the hypothesis that ligand activation of RXR suppresses host antiviral gene expression, we performed gene expression assays by qPCR. RAW264.7 cells were pretreated with 9cRA or HX531 overnight and then transfected with polyI:C. Ligand activation of RXR by 9cRA dramatically induced RXR targets genes, Abcal and Abcg1. However, the activation of RXR significantly suppressed primary 
a

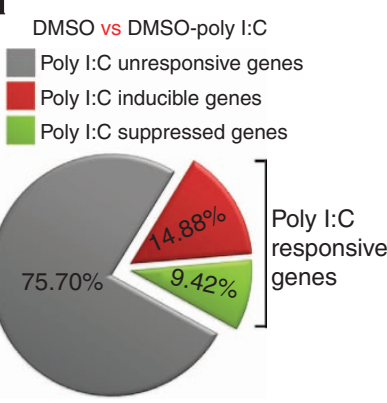

C
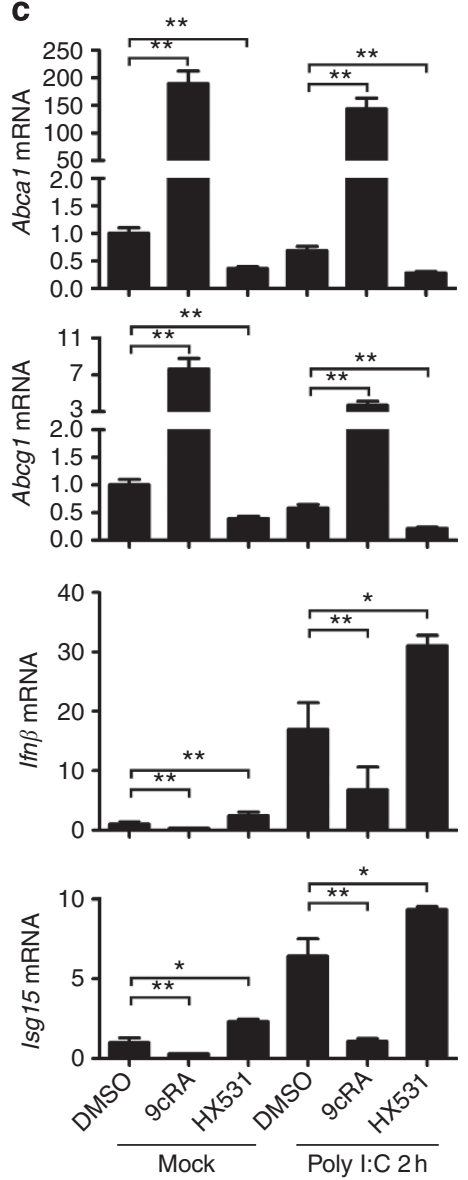

9cRA-inducible poly I:C-responsive genes

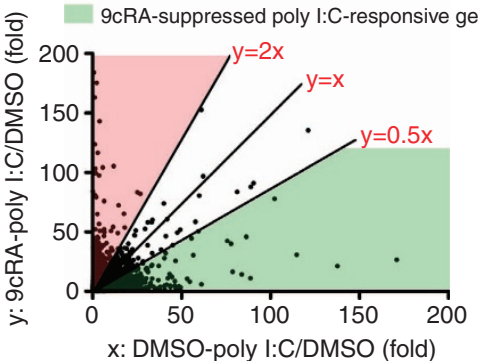

x: DMSO-poly I:C/DMSO (fold)

d
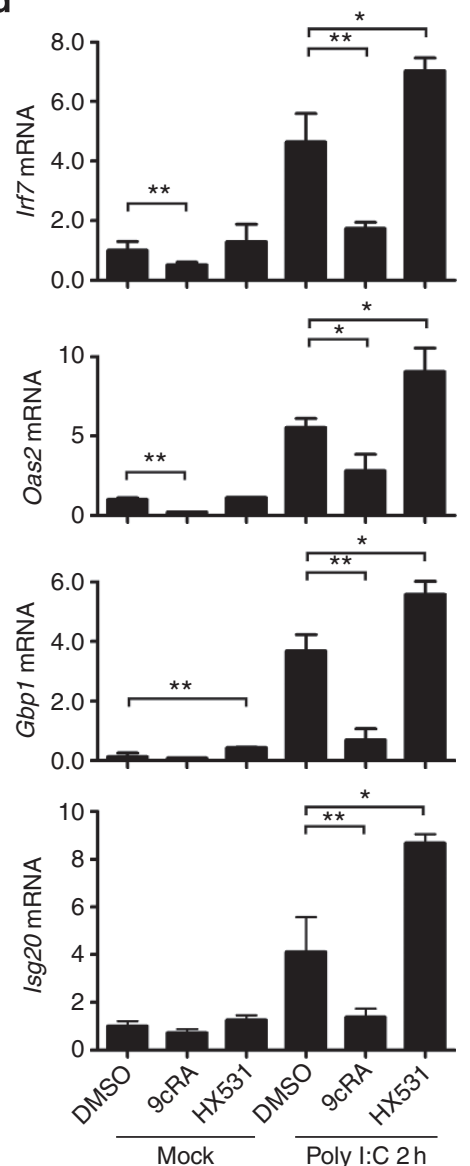

b

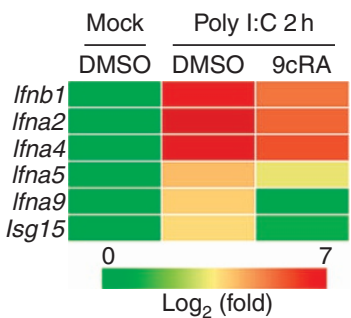

Figure 4 | Ligand activation of RXR inhibits type I IFN and ISGs. (a,b) RAW264.7 cells were pretreated with DMSO or 9cRA (100 nM) for $16 \mathrm{~h}$ and subsequently transfected with $1 \mu \mathrm{g} \mathrm{ml} \mathrm{m}^{-1}$ polyl:C for $2 \mathrm{~h}$. RNA was extracted and gene expression profile was detected by Affimetrix430.2 Chip. (a) Polyl:Cresponsive genes which included polyl:C-inducible genes (DMSO-polyl:C/DMSO $>2.0$ ) and polyl:C-suppressed genes (DMSO-polyl:C/DMSO <0.5) were further analysed, the polyl:C-responsive genes can be divided into two groups, 9cRA-inducible polyl:C-responsive genes ((9cRA-polyl:C/DMSO)/(DMSOpolyl:C/DMSO) > 2.0) and 9cRA-suppressed polyl:C-responsive genes ((9cRA-polyl:C/DMSO)/(DMSO-polyl:C/DMSO)<0.5). (b) Multiple type I IFN genes were 9cRA-suppressed polyl:C-responsive genes. (c) RAW264.7 cells were pretreated with DMSO, 9cRA (100 nM), or HX531(1 $\mu$ M) for $16 \mathrm{~h}$ and subsequently transfected with $1 \mu \mathrm{g} \mathrm{ml}{ }^{-1}$ polyl:C. RNA was collected $2 \mathrm{~h}$ after transfection, the mRNA expression of RXR target genes Abca1, Abcg1 and primary antiviral genes Ifn $\beta$, Isg15 were measured by qPCR. (d) RAW264.7 cells were pretreated as described in c, mRNA expression of ISGs with known antiviral activity, Irf7, Oas2, Gbp1 and Isg20 were quantified by qPCR. Data of $\mathbf{c}, \mathbf{d}$ are shown as mean \pm s.d. $(n=3)$ of one representative experiment, similar results were obtained in three independent experiments. ${ }^{\star} P<0.05$ and ${ }^{\star \star} P<0.01$ (Student's $t$-test).

antiviral genes, Ifn $\beta$ and Isg15 within $2 \mathrm{~h}$ after polyI:C transfection (Fig. 4c). Expression of ISGs with antiviral activity, such as Gbp1, Oas2, Irf7 and Isg20, was also suppressed in 9cRA-treated cells (Fig. 4d). RXR antagonist, HX531, significantly suppressed $A b c a 1$ and $A b c g 1$ expression while upregulated the expression of the antiviral genes tested (Fig. 4c,d). Interestingly, 9cRA and HX531 treatment also modulated the basal expression of some of these genes, suggesting that the activation of RXR by endogenous ligands during normal physiological states may suppress antiviral gene activation (Fig. 4c,d).

Upregulation of type I IFN in Rxra - / - BMMs. In addition to RAW264.7 cell lines, we observed similar results in immortalized J2 BMMs. 9cRA treatment suppressed If $n \beta$ and If $n \alpha 4$ expression, while HX531 increased Ifn $\beta$ expression in polyI:C-transfected J2 
a

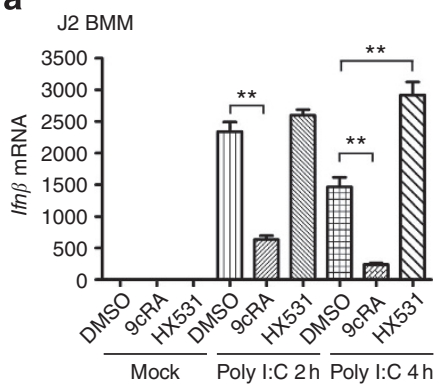

b

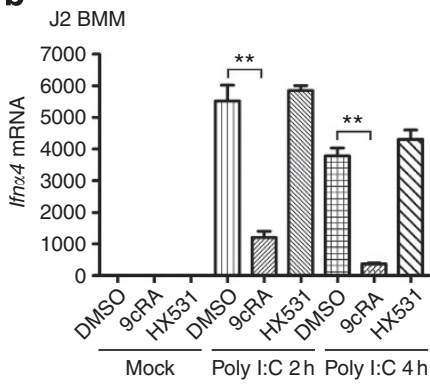

C

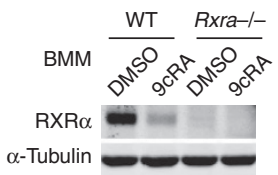

d

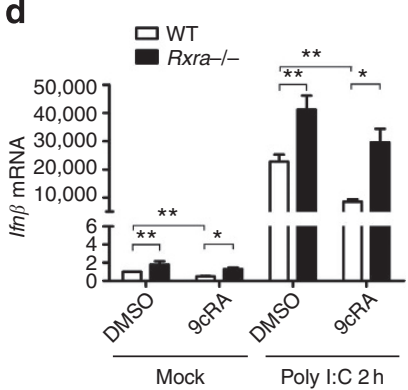

e

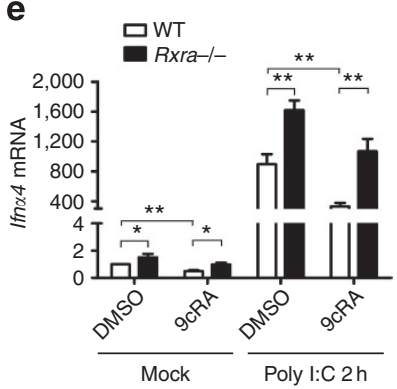

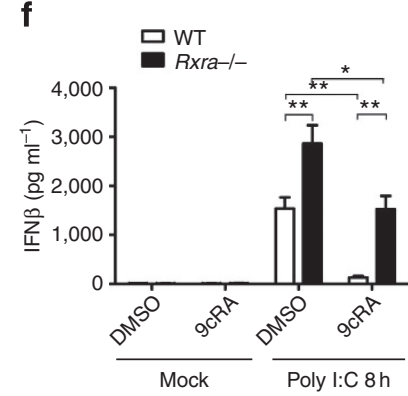

Figure 5 | RXR $\alpha$ negatively regulates type I IFN expression in immortalized and primary BMMs. (a,b) J2 BMMs were pretreated with DMSO, 9cRA $(100 \mathrm{nM})$ or $\mathrm{HX531}(1 \mu \mathrm{M})$ for $16 \mathrm{~h}$ and subsequently transfected with $1 \mu \mathrm{g} \mathrm{ml} \mathrm{l}^{-1}$ polyl:C for indicated time. The mRNA expression of Ifn $\beta$ (a) and Ifn $\alpha 4$ (b) were measured by qPCR. (c) Immunoblot of RXR $\alpha$ in the WT and Rxra - / - BMMs that pretreated with DMSO or 9cRA (100 nM) for 24h. (d-f) WTand Rxra - / - BMMs pretreated with DMSO or 9 cRA $(100 \mathrm{nM})$ for $24 \mathrm{~h}$, then the cells were transfected with $1 \mu \mathrm{g} \mathrm{ml} \mathrm{m}^{-1}$ polyl:C for $2 \mathrm{~h}$. Ifn $\beta$ (d) and Ifn $\alpha 4$ (e) mRNA expression were measured by qPCR. IFN $\beta$ protein level were detected by enzyme-linked immunosorbent assay after $8 \mathrm{~h}$ polyl:C transfection. Data of $\mathbf{a}, \mathbf{b}$ and $\mathbf{d}-\mathbf{f}$ are shown as mean \pm s.d. $(n=3)$ of one representative experiment, similar results were obtained in three independent experiments. ${ }^{\star} P<0.05$ and ${ }^{\star \star} P<0.01$ (Student's $t$-test). Data of $\mathbf{c}$ are representative of three independent experiments. See also Supplementary Fig. 7 of uncropped blot of $\mathbf{c}$

BMMs (Fig. 5a,b). Furthermore, we tested type I IFN expression in WT and Rxra-/- primary BMMs. No RXR $\alpha$ could be detected in the lys-Cre-expressed Rxra-/- BMMs (Fig. 5c). 9cRA inhibited basal IFN $\beta$ and IFN $\alpha 4$ expression, while higher basal IFN $\beta$ and IFN $\alpha 4$ were detected in Rxra-/- BMMs (Fig. 5d,e). PolyI:C transfection induced IFN $\beta$ and IFN $\alpha 4$ in both WT and Rxra-/- BMMs. However, 9cRA significantly suppressed the induction of IFN $\beta$ and IFN $\alpha 4$ in WT BMMs, but not in $R x r a-/-$ BMMs (Fig. 5d,e). Induction of IFN $\beta$ and IFN $\alpha 4$ was also higher in Rxra-/- BMMs than in WT BMMs (Fig. 5d,e). Consistently, 9cRA treatment dramatically suppressed IFN $\beta$ protein level in WT BMMs whereas Rxra-/- BMMs secreted more IFN than in WT BMMs (Fig. 5f). Interestingly, 9cRA also significantly suppressed IFN $\beta$ production in Rxra - / - BMMs (Fig. 5f), suggesting that ligand activation of $\operatorname{RXR} \beta$ and $\operatorname{RXR} \gamma$ might also downregulate IFN $\beta$ production in primary BMMs.

Taken together, these genetic and pharmacological studies provide evidence that RXR activation negatively regulates antiviral genes including type I IFN in macrophages.

RXR $\alpha$ inhibits type I IFN independent of IFNAR signalling. Generally, viral infections directly trigger expression of primary antiviral genes such as IFN $\beta$ and ISG15, and induction of IFN $\beta$ amplifies expression of antiviral effectors through autocrine or paracrine action ${ }^{27}$. To investigate the mechanism for the suppression of type I IFN by ligand activation of RXR, we used IFN $\alpha \beta$ receptor deficient BMMs (Ifnar $1-/-$ ) to test if ligand activation of RXR regulated primary type I IFN production or IFNAR downstream signalling-dependent type I IFN production positive feedback. Therefore, we pretreated Ifnar 1 - / - BMMs with 9cRA and HX531 and activated them by polyI:C transfection, expression of Ifn $\beta$, Isg15, and some ISGs were measured by qPCR. Ligand activation of RXR by 9cRA and LG268 significantly downregulated polyI:C-induced Ifn $\beta$ and Isg15 in Ifnar1-/- BMMs (Fig. 6a,b and Supplementary Fig. 4a,b). No induction of Oas2, Gbp1, Irf7 and Isg20 was observed in polyI:C-activated Ifnar1-/- BMMs (Supplementary Fig. 4c), which support that induction of these ISGs is type I IFN-dependent. These results suggest that ligand activation of RXR modulates the expression of a specific set of primary antiviral genes including IFN $\beta$ independent of IFNAR signalling, though we cannot exclude that RXR regulates IFNAR downstream signalling simultaneously.

We took the advantage of the IFN $\beta$ promoter luciferase reporter (IFN $\beta$-luc) to test if 9cRA suppressed IFN $\beta$ transcriptionally or post-transcriptionally. We found that $9 \mathrm{cRA}$ treatment significantly suppressed the IFN $\beta$-luc activity triggered by transfection of polyI:C (Fig. 6c), which suggests that ligand activation of RXR inhibits IFN $\beta$ transcription. VSV triggered host cell to produce type I IFN mainly through RIG-I and TRIF pathways, overexpression of the components of RIG-I and TRIF pathways can facilitate the host cells to clear the infected VSV. In HEK293T cells, more VSV-infected cells can be observed after overexpression of hRXRa, similar to the results with RAW264.7 (Fig. 6d). Overexpression of RIG-I, IPS1, TBK1 or IRF1 facilitated host cells to clear VSV; however, co-expression of $h R X R \alpha$ with these genes attenuated VSV clearance by host cells (Fig. 6e). Considering that transfection of RIG-I, IPS1, TBK1 or IRF1 triggered type I IFN in HEK293T cells, these data not only support the role of $\operatorname{RXR} \alpha$ in the increase of host susceptibility to VSV infection by type I IFN suppression, but also suggest RXR $\alpha$ potentially regulates type I IFN production downstream of TBK1.

Activation of RXR inhibit $\beta$-catenin nuclear translocation. Transfected polyI:C or infected VSV could be recognized by 
a

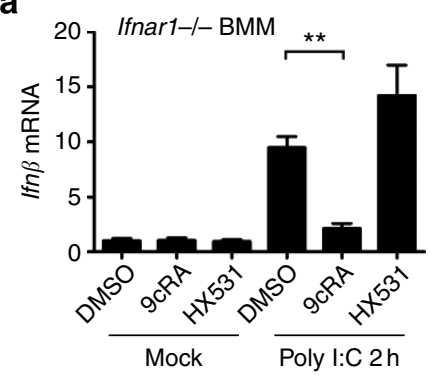

b

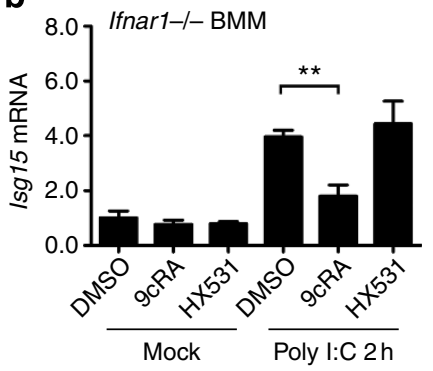

C

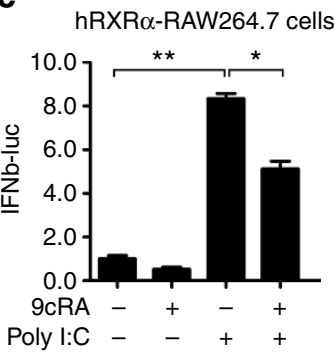

d

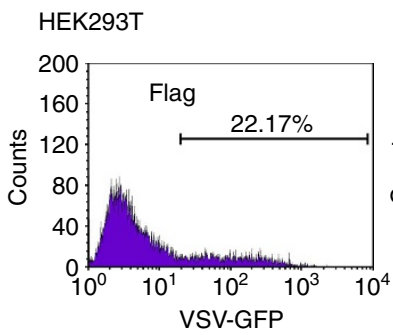

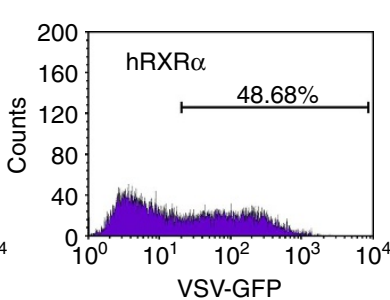

e

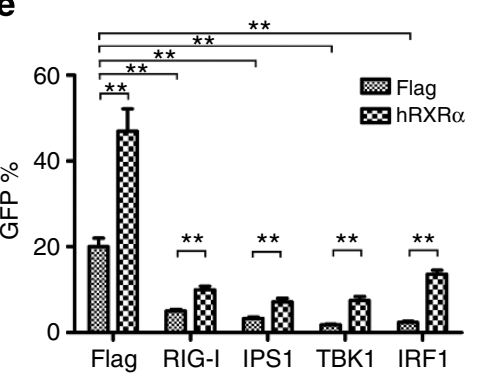

Figure 6 | RXR $\alpha$ inhibits type I IFN transcription independent of IFNAR signalling. (a,b) Ifnar - / - BMMs were pretreated with DMSO, 9cRA (100 nM) or HX531 $(1 \mu \mathrm{M})$ for $24 \mathrm{~h}$ and transfected with $1 \mu \mathrm{g} \mathrm{ml} \mathrm{m}^{-1}$ polyl:C. If $\beta$ and Isg15 mRNA were quantified by qPCR. Data are shown as mean $\pm \mathrm{s}$.d. ( $n=3$ ) of one representative experiment, similar results were obtained in three independent experiments, ${ }^{\star \star} P<0.01$ (Student's $t$-test). (c) hRXR $\alpha$-stably transfected RAW264.7 cells were transfected with IFN $\beta$ promoter luciferase reporter (IFN $\beta$-luc) and treated with DMSO or $9 \mathrm{cRA}(100 \mathrm{nM}$ ) for $9 \mathrm{~h}$ before stimulation with polyl:C $\left(5 \mu \mathrm{g} \mathrm{ml}^{-1}\right)$. The lysates were collected $20 \mathrm{~h}$ after polyl:C stimulation and luciferase reporter activity was measured by luminescence assay. RLU is represented as mean \pm s.d. $(n=6)$ and is representative of three experiments. ${ }^{\star} P<0.05$ and ${ }^{* \star} P<0.01$ (Student's $t$-test). (d) Flag or hRXR $\alpha$ vector was transfected into HEK293T cells for $24 \mathrm{~h}$, subsequently the transfected cells were infected by VSV-GFP $(\mathrm{MOI}=0.01)$ for $8 \mathrm{~h}$, fluorescence-activated cell sorting (FACS) assay was performed to detect the GFP-positive cells as VSV-infected cells. Data are representative of three independent experiments.

(e) Flag or hRXR $\alpha$ was co-transfected with RIG-I and TLR3 pathway components for $24 \mathrm{~h}$ into 293T cells, subsequently the transfected cells were infected by VSV-GFP $(\mathrm{MOI}=0.01)$ for $8 \mathrm{~h}$, FACS assay was performed to detect the VSV virus in the cells. The ratio of GFP-positive cell is represented as mean \pm s.d. $(n=3)$ and is representative of three experiments, $P<0.01$ (Student's $t$-test).

RIG-I and TLR3. RIG-I and TLR3 recruit the adaptor molecule IPS1 and TRIF, respectively. Both IPS1 and TRIF facilitate TBK1 and IRF3 phosphorylation, which leads to IRF3 dimerization and translocation into nucleus to drive IFN $\beta$ transcription ${ }^{24-26}$. RIG-I and TRIF pathway components triggered IFN $\beta$ promoter activity in HEK293T cells. However, the co-transfection of hRXR $\alpha$ significantly suppressed the IFN $\beta$ promoter activity activated by TRIF, RIG-I, IPS1, TBK1 and IRF3 (Fig. 7a). Considering that if hRXR $\alpha$ only regulates the expression or activity of RIG-I, IPS1 or TBK1, IFN $\beta$ promoter activity activated by the overexpression of IRF3 should not be affected. Therefore, these data suggest that RXR inhibits IFN $\beta$ downstream of IRF3, for example, IFN $\beta$ enhanceosome, although we cannot exclude the possibility that RXR concurrently regulates IFN $\beta$ production upstream of IRF3.

$\beta$-catenin, as a co-activator in the pathway for IRF3-mediated production of type I IFN, regulates the host antiviral response against Listeria monocytogenes, VSV, Sendai virus and HSV-1 (refs 28-31). Consistent with these studies, co-transfection with $\beta$-catenin significantly upregulated the IFN $\beta$ promoter activity activated by TRIF, RIG-I, IPS1, TBK1 and IRF3 (Fig. 7b). We hypothesized that hRXR $\alpha$ inhibits IFN $\beta$ enhanceosome by regulating $\beta$-catenin activity. By transfecting an efficient short interfering RNA (siRNA) to interfere with endogenous $\beta$-catenin in J2 BMMs, we found that polyI:C-triggered IFN $\beta$ expression was attenuated after the knockdown of $\beta$-catenin, and the inhibition of IFN $\beta$ induction by 9cRA and LG268 was almost abolished by the siRNA targeting $\beta$-catenin (Fig. 7c). Since activated $\beta$-catenin enters into the nucleus and forms a complex to drive IFN $\beta$ expression 28,29 , we compared the nuclear $\beta$-catenin between DMSO- and RXR agonists-treated macrophages. Both
polyI:C transfection and VSV infection did not change cytoplasmic $\beta$-catenin levels but increased nuclear $\beta$-catenin significantly. 9cRA and LG268 treatment suppressed $\beta$-catenin nuclear translocation compared with DMSO-treated controls (Fig. 7d,e and Supplementary Fig. 5). These results showed that RXR inhibited $\beta$-catenin translocation and led to the downregulation of type I IFN expression. In addition, by using the $\beta$-catenin reporter system ${ }^{42}$, we found that 9cRA and LG268 treatment inhibited the transcriptional activity of $\beta$-catenin in RAW264.7 cells, which was consistent with that ligand activation of RXR blocked $\beta$-catenin nuclear translocation (Supplementary Figs 6 and 7). Transfection of polyI:C significantly increased the binding of $\beta$-Catenin to Ifnb1 promoter region (Fig. $7 \mathrm{f}$ ); however, ligand activation of RXR by 9cRA or LG268 attenuated the binding between $\beta$-catenin and Ifnb1 promoter triggered by polyI:C transfection (Fig. $7 \mathrm{f}$ ). In summary, our data along with our published data showing that IRF3 downregulates RXR expression, illustrating that IRF3 not only activates antiviral gene expression directly, but also indirectly through the alleviation the RXR- $\beta$-catenin-dependent suppressive pathway to achieve optimal antiviral immune response (Fig. $7 \mathrm{~g}$ ).

\section{Discussion}

$\mathrm{RXR} \alpha$ is a key player in the cellular and whole-body metabolic and immune processes, and our previous study has suggested that the downregulation of $\mathrm{RXR} \alpha$ expression during viral infections would alter drug metabolisms leading to metabolic diseases such as Reye's Syndrome ${ }^{18}$. In our current study, we demonstrated that the downregulation of $\mathrm{RXR} \alpha$ is an innate immune defence 
a
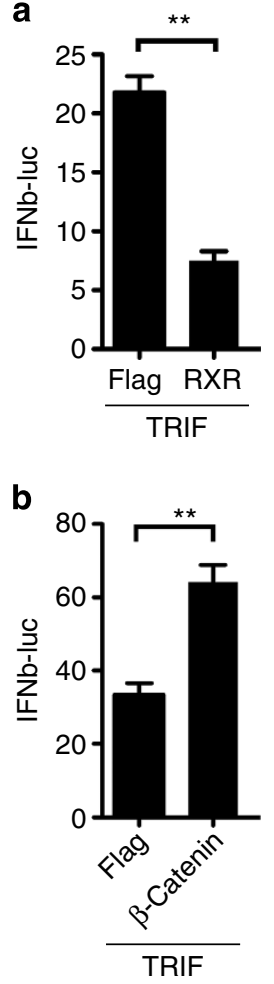
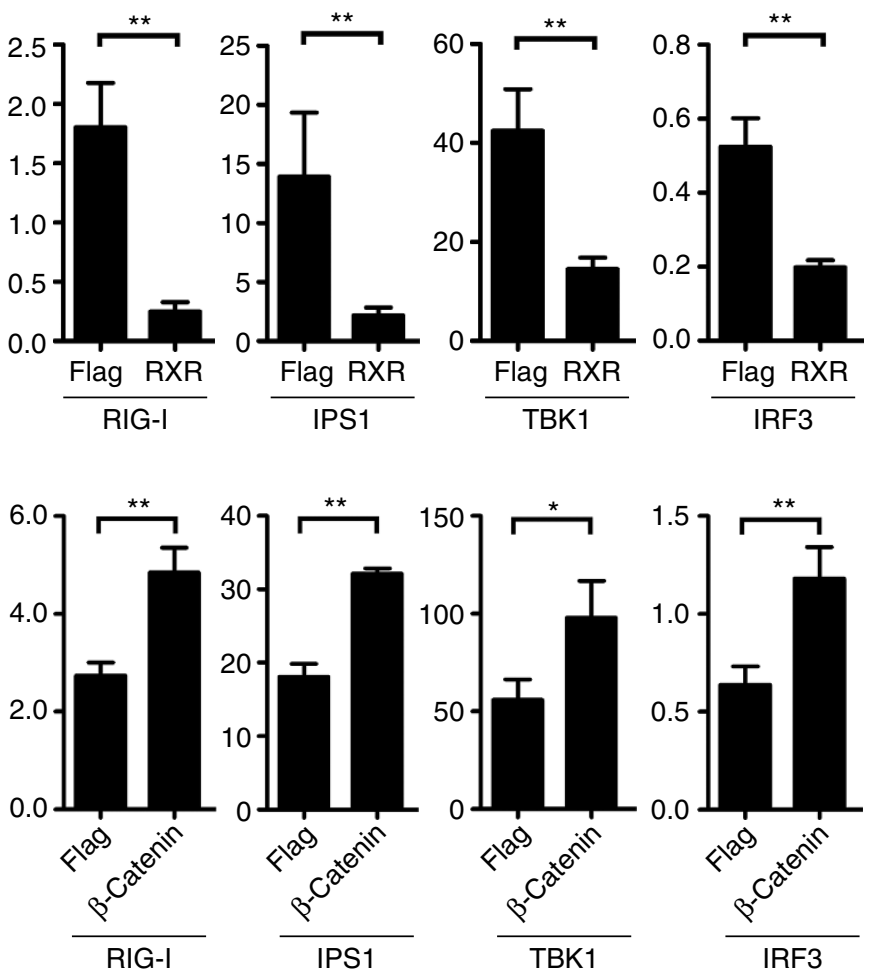

d

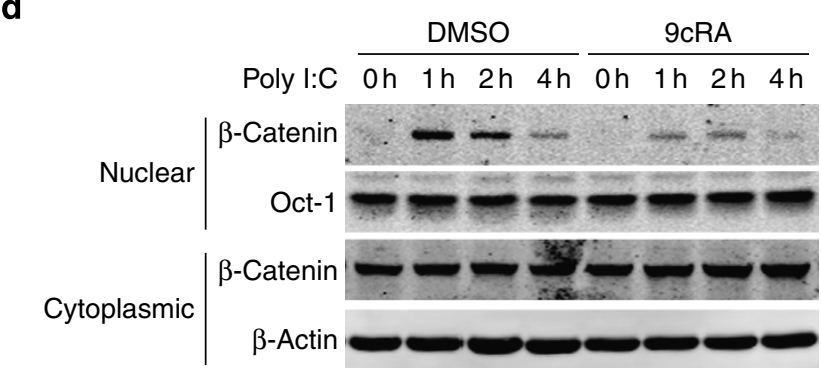

e

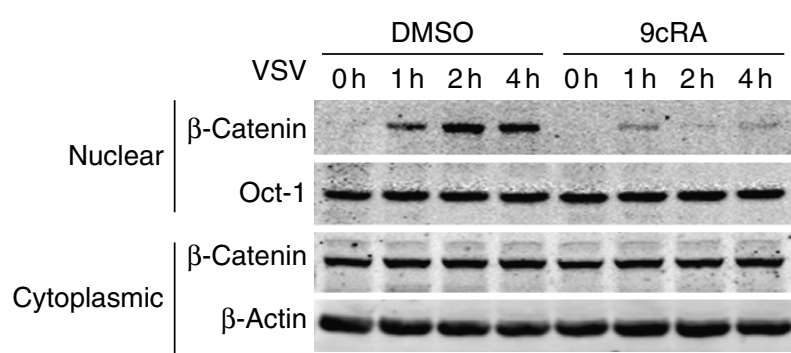

$\mathbf{9}$

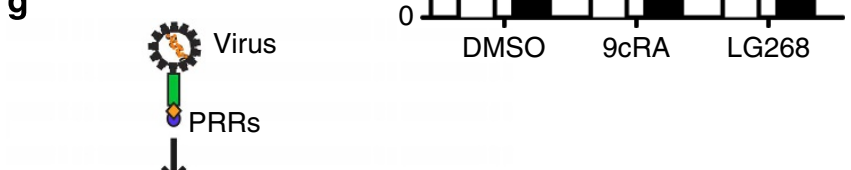

C

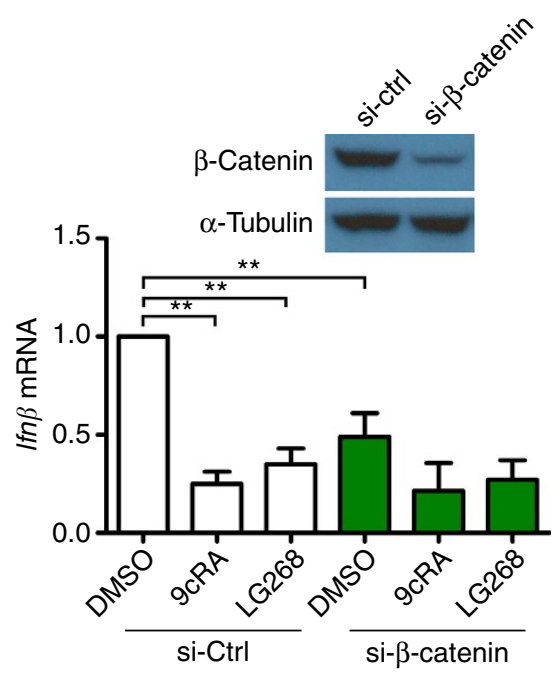

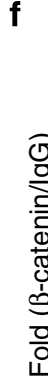

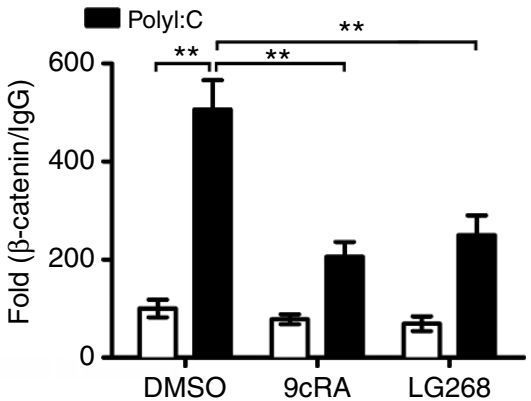

Figure 7 | Ligand activation of RXR $\boldsymbol{\alpha}$ inhibits $\boldsymbol{\beta}$-catenin nuclear translocation. (a,b) IFN $\beta$-luc reporter were co-transfected with flag or hRXR $\alpha$, and indicated components of RIG-I and TLR3 signalling pathway into 293 T cells for $24 \mathrm{~h}$, the luciferase activity of the lysate was detected (a). IFN $\beta$-luc reporter were co-transfected with flag or $\beta$-catenin, and indicated components of RIG-I and TLR3 signalling pathway into 293 T cells for $24 \mathrm{~h}$, the luciferase activity of the lysate was detected (b). IFN $\beta$-luc activity is represented as mean \pm s.d. $(n=6)$ and is representative of three experiments. ${ }^{\star} P<0.01$ (Student's $t$-test). (c) RAW264.7 cells were transfected with $20 \mathrm{nM}$ control siRNA (si-Ctrl) or siRNA targeting $\beta$-catenin (si- $\beta$-catenin). Twenty-four hours post transfection, the cells were treated with DMSO, 9cRA $(100 \mathrm{nM})$ or LG268 $(100 \mathrm{nM})$ for another $16 \mathrm{~h}$, and then these cells were transfected with $1 \mu \mathrm{g} \mathrm{ml}{ }^{-1}$ polyl:C for $2 \mathrm{~h}$, If $\beta$ mRNA expression were measured by qPCR. $\beta$-catenin protein level was quantified by immunoblot after the cells being transfected with siRNA for $24 \mathrm{~h}, \boldsymbol{\alpha}$-tubulin was shown as a loading control. (d,e) J2 BMMs were pretreated with DMSO or 9cRA (100 nM) for $16 \mathrm{~h}$, and then the cells were transfected with $3 \mu \mathrm{g} \mathrm{ml} \mathrm{I}^{-1}$ polyl:C (d) or infected by $1 \mathrm{MOI}$ VSV (e) for indicated time. Nuclear and cytoplasmic $\beta$-catenin were detected by immunoblot, Oct1 and $\beta$-actin were shown as loading controls. Data are representative of three independent experiments. (f) J2 BMMs were pretreated with DMSO, 9cRA $(100 \mathrm{nM})$ or LG268 $(100 \mathrm{nM})$ for $16 \mathrm{~h}$, and then the cells were transfected with $3 \mu \mathrm{g} \mathrm{ml}{ }^{-1}$ polyl:C for $2 \mathrm{~h}$, the binding of Ifnb1 promoter region with $\beta$-catenin (or IgG) was detected by ChIP-qPCR. The data was shown as fold change of $\beta$-catenin/lgG. Data of $\mathbf{c}$ and $\mathbf{f}$ are shown as mean \pm s.d. ( $n=3$ ) of one representative experiment, similar results were obtained in three independent experiments, ${ }^{\star \star} P<0.01$ (Student's $t$-test). Data of $\mathbf{d}$,e are representative of three independent experiments. See also Supplementary Fig. 7 of uncropped blot of $\mathbf{d}, \mathbf{e}$. (g) Working model for RXR $\alpha$ regulating host antiviral responses. Dash line: indirect regulation. 
mechanism important for host against viral infections. As RXR agonists and antagonists are increasingly used in clinics, our studies raises an open question about the potential differential effects of these drugs on patients with or without viral infections.

Previous studies have indicated that RXR heterodimers control host against Listeria monocytogenes and Mycobaterium tuberculosis infection 22,23 . A recent study suggested that $\mathrm{RXR} \alpha$ is an important factor in the gene network involved in host defence against Leishmania donovani ${ }^{43}$. In addition to controlling host immune responses to bacterial and parasitic infections, we have shown here that $\mathrm{RXR} \alpha$ overexpression or ligand activation increases host cell susceptibility to VSV, MHV68 and HSV-1 infection. RXR $\alpha$-deficient or RXR $\alpha$ antagonist-treated cells were more resistant to these viral infections. Moreover, RXR agonisttreated mice were more susceptible to HSV-1 infection. These results suggest that RXR signalling negatively regulates host antiviral response, and the downregulation of $\mathrm{RXR} \alpha$ benefits the host cellular immunity against viral infections. This study has described a unique crosstalk between host antiviral response and nuclear receptor RXR, suggesting that NRs may differentially affect host innate immunity against different pathogens.

Type I IFN and its downstream ISGs are critical for host defence against viruses ${ }^{27}$. Our microarray analysis and qPCR data indicated that multiple type I IFN genes and ISGs were suppressed in RXR agonist-treated macrophages. There was also increased basal and polyI:C-induced IFN $\beta$ expression in Rxra - / - BMMs than WT BMMs, providing the evidence that RXR signalling negatively regulates host antiviral response by inhibiting type I IFN and ISGs expression. Since ligand activation of RXR inhibits IFN $\beta$ promoter activity independent of IFN $\alpha \beta$ receptor and $\operatorname{RXR} \alpha$ might suppress IFN $\beta$ transcription downstream of the IRF3 transcription factor, we hypothesized that $\operatorname{RXR} \alpha$ regulates the components of IFN $\beta$ enhanceosome. $\beta$-catenin has been reported as a co-activator of IFN $\beta$ enhanceosome during viral and bacterial infection ${ }^{29-31}$. IFN $\beta$ reporter assays also suggest that $\beta$-catenin is a potential target to be regulated by RXR signalling. Ligand activation of RXR inhibits the transcription activity of $\beta$-catenin in macrophages, which is consistent with the previous study performed in HEK293 cells ${ }^{44}$. Knockdown of $\beta$-catenin almost abolished the RXR-dependent suppressive effect on IFN $\beta$ induction by polyI:C. By detecting the cytoplasmic and nuclear $\beta$-catenin, we observed that ligand activation of RXR suppressed nuclear $\beta$-catenin, while it had no effect on cytoplasmic $\beta$ catenin. Ligand activation of RXR also attenuated the binding of $\beta$-Catenin to Ifnb1 promoter. These data suggested that RXR could suppress $\beta$-Catenin nuclear translocation and subsequent type I IFN induction. However, the detail for the regulation of $\beta$ catenin by RXR needs to be further investigated, which is likely through an indirect mechanism as our preliminary results showed that the suppression of $\beta$-catenin nuclear translocation required overnight RXR agonist treatment and was also sensitive to new protein synthesis inhibitor, cycloheximide.

Our study supports a model by which modulation of RXR controls host antiviral immunity. During the absence of infection, RXR is constitutively expressed and can be activated by multiple endogenous RXR ligands, such as 9cRA, docosahexaenoic acid, oleic acid and phytanic acid, to suppress basal and inducible IFN expression. However, during viral infection, the host cell downregulates $\mathrm{RXR} \alpha$ expression in an IRF3-dependent manner to achieve optimal IFN expression. The major function of IRF3 is known to directly bind to the promoter region of primary type I IFN genes such as IFN $\beta$ to initiate IFN response. Our study, therefore, reveals an indirect function of IRF3 in IFN induction through relieving RXR-mediated suppression of IFN genes. After infection, to avoid excess type I IFN production, RXR expression level is restored by host cells and exerts its suppressive function on the type I IFN induction in the presence of the RXR endogenous ligands. Therefore, RXR not only maintains the basal type I IFN and modulates the host antiviral response, but also regulates the resolution of infection.

Retinoids and rexinoids are frequently used in the clinical setting as treatment for cancer, inflammatory skin diseases and acne $e^{7,11,15}$. Accutane and Isotretinoin have been approved to treat acne and cancer ${ }^{37-39}$. Targretin has been used in therapy for the treatment of cutaneous T-cell lymphoma and is in clinical trials for the treatment of breast and lung cancer ${ }^{15}$. In addition to their applications in cancer, rexinoids are being tested in the treatment of diabetes, obesity, atherosclerosis and other cardiovascular indications and inflammatory diseases ${ }^{3,11}$. On the other hand, treatment with rexinoids or retinoids raises plasma triglyceride levels, suppresses the thyroid hormone axis and induces hepatomegaly ${ }^{11,15}$. Our results show that retinoids or rexinoids treatment increases susceptibility to HSV-1 infection in mice and support clinical reports of association of Isotretinoin with herpetic infections ${ }^{40,41}$. However, further investigation is needed to evaluate the overall impact of the RXR agonists on virally infected patients. Nonetheless, our data present the potential application of RXR antagonist in treatment of viral infectionrelated diseases.

\section{Methods}

Mice and reagents. C57BL/6 male mice (6-8 weeks of age) and Ifnar 1 - / - mice were from the Jackson Laboratory. All mice experiments were performed in accordance with guidelines from the University of California, Los Angeles, Institutional Animal Care and Use Committee. HEK293T and RAW264.7 cells were purchased from ATCC and cultured in Dulbecco's modified Eagle's medium (DMEM) with $10 \%$ fetal bovine serum (FBS) and 1\% penicillin/streptomycin. $\mathrm{RXR}^{\mathrm{fl} / \mathrm{fl}}(\mathrm{WT})$ and LysM-Cre ${ }^{+} / \mathrm{RXR}^{\mathrm{fl} / \mathrm{fl}}(\mathrm{Rxra}-/-)$ mice bone marrows were overnight shipped from Dr Mercedes Ricote's Lab (Spain) ${ }^{13}$. WT, Ifnar1 - / and Rxra-1- BMMs were differentiated as described previously ${ }^{45}$. WT and Rxra-/- F9 embryocarcinoma cells, as well as RXR-specific agonists AGN194204 and LG268 were obtained from Dr Peter Tontonoz's Lab (University of California, Los Angeles). HX531 was obtained from Dr Hiroyuki Kagechika's lab (Tokyo Medical and Dental University). 9cRA and 13cRA were purchased from Sigma-Aldrich. All compounds for in vitro treatment were solubilized in DMSO. PolyI:C and polydA:dT were from InvivoGen. Antibody against $\alpha$-tubulin was from Sigma-Aldrich. Anti-VSV-G (P5D4) and anti-Oct1 (C-21) antibodies were from Santa Cruz Biotechnology. Anti-RXR $\alpha$ (D6G10), anti- $\beta$-Actin (13E5) and anti- $\beta$-Catenin (D10A8) were from Cell Signaling Technology. Anti-GAPDH (GT239) was from GeneTex.

Stable cell lines and BMM immortalization. hRXR $\alpha$-flag, hRXR $\alpha \mathrm{K} 108 \mathrm{R}-\mathrm{flag}$ and $\mathrm{hRXR} \alpha \Delta \mathrm{EF}$-flag were cloned into $\mathrm{pBABE}$ retroviral vector to make WT or mutated $\mathrm{hRXR} \alpha \mathrm{pBABE}$ constructs. These constructs or $\mathrm{pBABE}-\mathrm{empty}$ vector was cotransfected with packaging vectors psiA into HEK293T cells for $48 \mathrm{~h}$. The viral supernatants were collected and used to transduce control or hRXR $\alpha$ (WT and its mutants) into RAW264.7 or F9 embryocarcinoma cells. The transduced cells were selected by puromycin $\left(2 \mu \mathrm{g} \mathrm{ml}^{-1}\right)$ for 2 weeks, and then the transformed cells were stable cell lines. For J2 virus-immortalized macrophages (J2 BMMs), a cell line transformed by retrovirus expressing $\mathrm{v}$-raf and c-myc, was established (called GG2EE) and grown in RPMI1640 (10 mM HEPES PH7.8, 10\% FBS, 1\% Pen/ strep $)^{46,47}$. The supernatant containing J2 viruses was harvested and filtered through $0.22 \mu \mathrm{m}$ filter. Bone marrow cells were infected with the $\mathrm{J} 2$ virus and immortalized as J2 BMMs.

Viral mimics and siRNA transfection. For activating cells by transfection with polyI:C and polydA:dT in a six-well plate, $5 \mu \mathrm{l}$ of Lipofectamine 2000 (Life Technolgies) was diluted in $200 \mu \mathrm{l}$ Opti-MEM for $5 \mathrm{~min}$. PolyI:C or polydA:dT $(2 \mu \mathrm{g})$ were diluted in $200 \mu \mathrm{l}$ Opti-MEM. Mixed the above Lipofectamine 2000 solution and polyI:C (or polydA:dT) solution, and incubated in room temperature for $15 \mathrm{~min}$. Subsequently, the mixed solution was added into the cells with $1.6 \mathrm{ml}$ fresh medium. Jet-PEI (Polyplus Transfection) was used to transfect plasmids into HEK293T cells according to the manufacturer's instructions. siRNA target mouse $\beta$-catenin (SMARTpool: siGENOME Ctnnbl siRNA) and its control were purchased from Dharmacon. RAW264.7 cells were transfected with $20 \mathrm{nM}$ control or $\beta$-catenin by using INTERFERin (Polyplus Transfection) according to the manufacturer's instructions. 
Ligand activation and viral Infection. RAW264.7 cells were plated in a six-well format at $6 \times 10^{5}$ and treated with DMSO, RXR agonists or RXR antagonist for 16-24h. Replication competent VSV-GFP was graciously provided by Dr Glen Barber and MHV68-luc was from Dr Ren Sun Lab (University of California, Los Angeles). Viruses were propagated as described previously ${ }^{48,49}$. Cells were infected with VSV (multiplicity of infection, MOI $=0.01$ ) in media for $1 \mathrm{~h}$ and replenished with DMEM with $1 \%$ FBS. Supernatants were collected between 12 and 16 h.p.i. MHV68-luc infection was quantified by firefly luciferase assay kit (Promega Inc.). VSV-G pseudotyped virus containing renilla luciferase reporter was a gift from Dr Benhur Lee (University of California, Los Angeles). Infections were performed similarly and quantified by renilla luciferase kit (Promega Inc.). Flow cytometry was performed to detect the VSV-GFP in HEK293T cells on FACScaliber (BD Biosciences) with standard compensation and the data was analysed using CellQuest (BD Biosciences).

Plaque assay. Standard plaque assay was performed on a monolayer of Vero cells. Briefly, Vero cells were seeded at $2.5 \times 10^{5}$ cells per well in standard 12 -well plates. Tenfold serial dilutions of VSV viral supernatants were made. Vero cells were infected with diluted supernatants for $1 \mathrm{~h}$ and resuspended in $1 \%$ FBS with $0.6 \%$ low-melt point agarose in DMEM overnight. The agarose was removed by suction and stained with $0.05 \%$ crystal violet, $40 \% \mathrm{EtOH}$, in PBS. HSV $-1(\mathrm{MOI}=0.25)$ and MHV68-luc $(\mathrm{MOI}=1)$ infections were done by a similar way. HSV-1 plaque assay took $60 \mathrm{~h}$ for plaques to form and were stained the same way.

RNA isolation, reverse transcrption and real time qPCR. Biological triplicates of RAW264.7 cells were pretreated with 9cRA $(100 \mathrm{nM})$ or HX531 $(1 \mu \mathrm{M})$ for $16 \mathrm{~h}$ and subsequently transfected with polyI:C $\left(1 \mu \mathrm{g} \mathrm{ml}^{-1}\right)$. Cells were lysed in Trizol and RNA was isolated by standard isopropanol precipitation. RNA was quantified by spectrometry. cDNA was generated using $1 \mu \mathrm{g}$ of RNA with IScript reverse transcription kit (BioRad) according to the manufacturer's instructions with either random hexamers or VSV N1 (5' GATAGTACCGGAGGATTGACGACTA-3') as primers. qPCR analysis was done using the iCycler thermocycler (Bio Rad). qPCR was performed by using Sensimix SYBR \& Fluorescein Kit (Bioline) according to the manufacturer's instructions. Expression values were normalized to L32 control and fold induction was normalized to untreated control by using $2^{-\triangle \Delta C t}$ Method $^{50}$. Gene primer sequences used were: Rxra: $5^{\prime}$-ATGGACACCA AACATTTCCTGC- $3^{\prime}$ and $5^{\prime}$-CCAGTGGAGAGCCGATTCC- $3^{\prime}$; VSV: $5^{\prime}$-GATA GTACCGGAGGATTGACGACTA- $3^{\prime}$ and $5^{\prime}$-CTTACCGAGCCTACCAAACT; Abcal: $5^{\prime}$-GCTTGTTGGCCTCAGTTAAGG-3' and $5^{\prime}$-GTAGCTCAGGCGT ACAGAGAT-3'; Abcg1: 5'-GGAGTACGGGGATCAGAACA-3' and 5'-AAAGA AACGGGTTCACATCG- $3^{\prime}$; Ifnb1: $5^{\prime}$-CAGCTCCAAGAAAGGACGAAC- $3^{\prime}$ and $5^{\prime}$-GGCAGTGTAACTCTTCTGCAT-3'; Ifna4: 5'-TGATGAGCTACTACTG GTCAGC- $3^{\prime}$ and $5^{\prime}$-GATCTCTTAGCACAAGGATGGC- ${ }^{\prime}$; Isg15: $5^{\prime}$-CAGGAC GGTCTTACCCTTTCC- $3^{\prime}$ and $5^{\prime}$-AGGCTCGCTGCAGTTCTGTAC- $3^{\prime}$; Gbp 1 $5^{\prime}$-CAGGGTAGACAATGGGCAGT- $3^{\prime}$ and $5^{\prime}$-CACAGTAGGCTGGAGCATG A- $3^{\prime}$; Oas2: $5^{\prime}$-GGTGAGGGTCCCATAACCTT- $3^{\prime}$ and $5^{\prime}$-AGTCCACCAGA GCCTGCTTA- $3^{\prime}$; Irf7: $5^{\prime}$-ACAGGGCGTTTTATCTTGCG- ${ }^{\prime}$ and $5^{\prime}$-TCCAAGC TCCCGGCTAAGT- $3^{\prime}$; Isg20: $5^{\prime}$-CCATGGACTGTGAGATGGTG- $3^{\prime}$ and $5^{\prime}$-CT CGGGTCGGATGTACTTGT-3'; L32: $5^{\prime}$-AAGCGAAACTGGCGGAAAC- $3^{\prime}$ and $5^{\prime}$-TAACCGATGTTGGGCATCAG-3'.

Assay of luciferase reporter gene expression. HEK293T or hRXR $\alpha$-RAW264.7 cells were co-transfected with a mixture of IFN $\beta$ firefly luciferase reporter plasmids (IFN $\beta$-luc), pRL-TK renilla luciferase plasmids and other indicated constructs by using Jet-PEI (Polyplus Transfection) according to the manufacturer's instructions. Luciferase activity was measured with the Dual-Luciferase Reporter Assay System (Promega Inc.) as described ${ }^{51,52}$. Data were normalized for transfection efficiency by division of firefly luciferase activity with renilla luciferase activity.

Nuclear and cytoplasmic protein separation and western blot. Cells were harvested and lysed with M-PER Protein Extraction Reagent (Pierce, Rockford, IL) supplemented with protease inhibitor cocktail (Calbiochem). Nuclear protein was extracted using NE-PER nuclear and Cytoplasmic Extraction Reagents (Pierce). Protein concentrations of the extracts were measured with bicinchoninic acid assay (Pierce) and equalized with the extraction reagent. Equal amounts of the extracts were used for immunoprecipitation, or loaded and subjected to SDS-polyacrylamide gel electrophoresis, transferred onto nitrocellulose membranes and then blotted as described previously ${ }^{51,52}$.

HSV-1 infection in vivo. The RXR agonists LG268, 9cRA and 13cRA were dissolved in DMSO $\left(25 \mu \mathrm{g} \mu \mathrm{l}^{-1}\right)$. DMSO or RXR $(1 \mu \mathrm{l})$ agonist solution was diluted in $100 \mu \mathrm{l}$ saline for each mice injection. WT C57/BL6 were injected i.p. with DMSO, LG268, 9cRA or 13cRA ( $25 \mu \mathrm{g}$ per mice) daily for 4 days $(n=5)$. The mice were infected with HSV-1(10 7 p.f.u. of Strain 17) on day 5. Mice were killed 5 d.p.i and the livers were harvested, homogenized in media and quantified for viral titres by standard plaque assay.
Affimetrix microarray. Gene expression profiling of RAW264.7 cells pretreated with DMSO or 9cRA and activated by polyI:C transfection was done on an Affymetrix 430.2 Chip by the UCLA Genotyping and Array.

ELISA and ChIP assay. IFN $\beta$ in culture supernatant was quantified with VeriKine Mouse Interferon Beta ELISA Kit (PBL interferon source) according to the manufacturer's instructions. Chromatin immunoprecipitation (ChIP) assays were performed according to the protocol of the ChIP assay kit (Magna ChIP HiSens kit, catalogue No. 17-10460, Millipore). $\beta$-catenin (D10A8) XP Rabbit mAb (\#8480) from Cell Signaling Technology was used for ChIP assay at the concentration of $2 \mu \mathrm{g}$ antibody per reaction. Primer pair specific for the Ifnbl promoter region has been described ${ }^{33}$. Ifnb1 promoter region in both input and immunoprecipitated genomic DNA was detected by qPCR. The percentage of Ifnbl promoter region immunoprecipitated by $\beta$-catenin or its isotype IgG relative to input DNA was calculated, the data was shown the enrichment of the Ifnbl promoter region by $\beta$-catenin.

\section{References}

1. Repa, J. J. et al. Regulation of absorption and ABC1-mediated efflux of cholesterol by RXR heterodimers. Science 289, 1524-1529 (2000).

2. Kastner, P. et al. Genetic analysis of RXR alpha developmental function: convergence of RXR and RAR signaling pathways in heart and eye morphogenesis. Cell 78, 987-1003 (1994)

3. Altucci, L., Leibowitz, M. D., Ogilvie, K. M., de Lera, A. R. \& Gronemeyer, H. RAR and RXR modulation in cancer and metabolic disease. Nat. Rev. Drug Discov. 6, 793-810 (2007).

4. Nagy, L., Szanto, A., Szatmari, I. \& Szeles, L. Nuclear hormone receptors enable macrophages and dendritic cells to sense their lipid environment and shape their immune response. Physiol. Rev. 92, 739-789 (2012).

5. Mangelsdorf, D. J. \& Evans, R. M. The RXR heterodimers and orphan receptors. Cell 83, 841-850 (1995).

6. Lefebvre, P., Benomar, Y. \& Staels, B. Retinoid X receptors: common heterodimerization partners with distinct functions. Trends Endocrinol. Metab. 21, 676-683 (2010).

7. Dawson, M. I. \& Xia, Z. The retinoid X receptors and their ligands. Biochim. Biophys. Acta 1821, 21-56 (2012).

8. Mangelsdorf, D. J. et al. Characterization of three RXR genes that mediate the action of 9-cis retinoic acid. Genes Dev. 6, 329-344 (1992).

9. Rowe, A., Eager, N. S. \& Brickell, P. M. A member of the RXR nuclear receptor family is expressed in neural-crest-derived cells of the developing chick peripheral nervous system. Development 111, 771-778 (1991).

10. Yu, V. C. et al. RXR beta: a coregulator that enhances binding of retinoic acid, thyroid hormone, and vitamin D receptors to their cognate response elements. Cell 67, 1251-1266 (1991)

11. Roszer, T., Menendez-Gutierrez, M. P., Cedenilla, M. \& Ricote, M. Retinoid X receptors in macrophage biology. Trends Endocrinol. Metab. 24, 460-468 (2013).

12. Ricote, M. et al. Normal hematopoiesis after conditional targeting of RXRalpha in murine hematopoietic stem/progenitor cells. J. Leukoc. Biol. 80, 850-861 (2006).

13. Nunez, V. et al. Retinoid X receptor alpha controls innate inflammatory responses through the up-regulation of chemokine expression. Proc. Natl Acad. Sci. USA 107, 10626-10631 (2010)

14. Mangelsdorf, D. J., Ong, E. S., Dyck, J. A. \& Evans, R. M. Nuclear receptor that identifies a novel retinoic acid response pathway. Nature 345, 224-229 (1990).

15. Perez, E., Bourguet, W., Gronemeyer, H. \& de Lera, A. R. Modulation of RXR function through ligand design. Biochim. Biophys. Acta 1821, 57-69 (2012).

16. Kempf, W., Kettelhack, N., Duvic, M. \& Burg, G. Topical and systemic retinoid therapy for cutaneous T-cell lymphoma. Hematol. Oncol. Clin. North Am. 17, 1405-1419 (2003).

17. Zhang, C. \& Duvic, M. Retinoids: therapeutic applications and mechanisms of action in cutaneous T-cell lymphoma. Dermatol. Ther. 16, 322-330 (2003).

18. Chow, E. K. et al. A role for IRF3-dependent RXRalpha repression in hepatotoxicity associated with viral infections. J. Exp. Med. 203, 2589-2602 (2006).

19. Joseph, S. B., Castrillo, A., Laffitte, B. A., Mangelsdorf, D. J. \& Tontonoz, P Reciprocal regulation of inflammation and lipid metabolism by liver $\mathrm{X}$ receptors. Nat. Med. 9, 213-219 (2003).

20. Na, S. Y. et al. Retinoids inhibit interleukin-12 production in macrophages through physical associations of retinoid X receptor and NFkappaB. J. Biol. Chem. 274, 7674-7680 (1999).

21. Wang, X., Allen, C. \& Ballow, M. Retinoic acid enhances the production of IL-10 while reducing the synthesis of IL-12 and TNF-alpha from LPSstimulated monocytes/macrophages. J. Clin. Immunol. 27, 193-200 (2007).

22. Joseph, S. B. et al. LXR-dependent gene expression is important for macrophage survival and the innate immune response. Cell 119, 299-309 (2004).

23. Liu, P. T. et al. Toll-like receptor triggering of a vitamin D-mediated human antimicrobial response. Science 311, 1770-1773 (2006). 
24. Takeuchi, O. \& Akira, S. Innate immunity to virus infection. Immunol. Rev. 227, 75-86 (2009).

25. Goubau, D., Deddouche, S. \& Reis, E. S. C. Cytosolic sensing of viruses. Immunity 38, 855-869 (2013).

26. Newton, K. \& Dixit, V. M. Signaling in innate immunity and inflammation. Cold Spring Harbor perspectives in biology 4, a006049 (2012).

27. Sadler, A. J. \& Williams, B. R. Interferon-inducible antiviral effectors. Nat. Rev. Immunol. 8, 559-568 (2008).

28. Rathinam, V. A., Sharma, S. \& Fitzgerald, K. A. Catenin' on to nucleic acid sensing. Nat. Immunol. 11, 466-468 (2010).

29. Yang, P. et al. The cytosolic nucleic acid sensor LRRFIP1 mediates the production of type I interferon via a beta-catenin-dependent pathway. Nat. Immunol. 11, 487-494 (2010).

30. Zhu, J., Coyne, C. B. \& Sarkar, S. N. PKC alpha regulates Sendai virusmediated interferon induction through HDAC6 and beta-catenin. EMBO J. 30, 4838-4849 (2011).

31. Gantner, B. N. et al. The Akt1 isoform is required for optimal IFN-beta transcription through direct phosphorylation of beta-catenin. J. Immunol. 189, 3104-3111 (2012).

32. Choi, S. J. et al. Negative modulation of RXRalpha transcriptional activity by small ubiquitin-related modifier (SUMO) modification and its reversal by SUMO-specific protease SUSP1. J. Biol. Chem. 281, 30669-30677 (2006).

33. Rochette-Egly, C. \& Chambon, P. F9 embryocarcinoma cells: a cell autonomous model to study the functional selectivity of RARs and RXRs in retinoid signaling. Histol. Histopathol. 16, 909-922 (2001).

34. Clifford, J., Chiba, H., Sobieszczuk, D., Metzger, D. \& Chambon, P. RXRalphanull F9 embryonal carcinoma cells are resistant to the differentiation, antiproliferative and apoptotic effects of retinoids. EMBO J. 15, 4142-4155 (1996).

35. Ebisawa, M. et al. Retinoid X receptor-antagonistic diazepinylbenzoic acids. Chem. Pharm. Bull. (Tokyo). 47, 1778-1786 (1999).

36. Negrete, O. A. et al. EphrinB2 is the entry receptor for Nipah virus, an emergent deadly paramyxovirus. Nature 436, 401-405 (2005).

37. Berg, W. J. et al. Up-regulation of retinoic acid receptor beta expression in renal cancers in vivo correlates with response to 13-cis-retinoic acid and interferonalpha-2a. Clin. Cancer Res. 5, 1671-1675 (1999).

38. Tsimberidou, A. M., Giles, F., Romaguera, J., Duvic, M. \& Kurzrock, R. Activity of interferon-alpha and isotretinoin in patients with advanced, refractory lymphoid malignancies. Cancer 100, 574-580 (2004).

39. Recchia, F. et al. Phase II randomized study of interleukin-2 with or without 13-cis retinoic acid as maintenance therapy in patients with advanced cancer responsive to chemotherapy. Anticancer Res. 25, 3149-3157 (2005).

40. Baxter, K. F. \& Cunliffe, W. J. Retinoid herpeticum. Clin. Exp. Dermatol. 26, 557-558 (2001)

41. Stetson, C. L., Butler, D. F. \& Rapini, R. P. Herpetic whitlow during isotretinoin therapy. Int. J. Dermatol. 42, 496-498 (2003).

42. Veeman, M. T., Slusarski, D. C., Kaykas, A., Louie, S. H. \& Moon, R. T. Zebrafish prickle, a modulator of noncanonical $\mathrm{Wnt} / \mathrm{Fz}$ signaling, regulates gastrulation movements. Curr. Biol. 13, 680-685 (2003).

43. Beattie, L. et al. A transcriptomic network identified in uninfected macrophages responding to inflammation controls intracellular pathogen survival. Cell Host Microbe 14, 357-368 (2013).

44. Xiao, J. H. et al. Adenomatous polyposis coli (APC)-independent regulation of beta-catenin degradation via a retinoid X receptor-mediated pathway. J. Biol. Chem. 278, 29954-29962 (2003).
45. Chin, A. I. et al. Involvement of receptor-interacting protein 2 in innate and adaptive immune responses. Nature 416, 190-194 (2002).

46. Palleroni, A. V., Varesio, L., Wright, R. B. \& Brunda, M. J. Tumoricidal alveolar macrophage and tumor infiltrating macrophage cell lines. Int. J. Cancer 49, 296-302 (1991).

47. Zhou, H., Imrich, A. \& Kobzik, L. Characterization of immortalized MARCO and SR-AI/II-deficient murine alveolar macrophage cell lines. Part. Fibre Toxicol. 5, 7 (2008).

48. Doyle, S. et al. IRF3 mediates a TLR3/TLR4-specific antiviral gene program. Immunity 17, 251-263 (2002).

49. Liu, S. Y., Sanchez, D. J., Aliyari, R., Lu, S. \& Cheng, G. Systematic identification of type I and type II interferon-induced antiviral factors. Proc. Natl Acad. Sci. USA 109, 4239-4244 (2012).

50. Schmittgen, T. D. \& Livak, K. J. Analyzing real-time PCR data by the comparative C(T) method. Nat. Protoc. 3, 1101-1108 (2008).

51. Ma, F. et al. MicroRNA-466l upregulates IL-10 expression in TLR-triggered macrophages by antagonizing RNA-binding protein tristetraprolin-mediated IL-10 mRNA degradation. J. Immunol. 184, 6053-6059 (2010).

52. Ma, F. et al. The microRNA miR-29 controls innate and adaptive immune responses to intracellular bacterial infection by targeting interferon-gamma. Nat. Immunol. 12, 861-869 (2011).

53. Mokrani, H., Sharaf el Dein, O., Mansuroglu, Z. \& Bonnefoy, E. Binding of YY1 to the proximal region of the murine beta interferon promoter is essential to allow CBP recruitment and $\mathrm{K} 8 \mathrm{H} 4 / \mathrm{K} 14 \mathrm{H} 3$ acetylation on the promoter region after virus infection. Mol. Cell Biol. 26, 8551-8561 (2006).

\section{Acknowledgements}

We thank David Sanchez for his editorial advice and Harvey Roy Herschman for his helpful discussions. This work was funded by NIH RO1 AI078389, AI056154, AI47868, and AI069120 grants, the Tumor Immunology Training Grant (5T32CA009120), the grant from the Spanish Ministry of Economy and Competitiveness (SAF2012-31483) and the Medical Scientist Training Program.

\section{Author contributions}

F.M., S.-Y.L. and G.C. designed the study and wrote the manuscript; S.-Y.L, F.M., B.R., N.A., B.L. and V.N. performed the experiments; H.K., P.T. and M.R. provided reagents for this study; and all authors read and approved the manuscript.

\section{Additional information}

Accession codes: Microarray data was deposited in GEO (Accession number: GSE62107)

Supplementary Information accompanies this paper at http://www.nature.com/ naturecommunications

Competing financial interests: The authors declare no competing financial interests.

Reprints and permission information is available online at http://npg.nature.com/ reprintsandpermissions/

How to cite this article: Ma, F. et al. Retinoid X receptor $\alpha$ attenuates host antiviral response by suppressing type I interferon. Nat. Commun. 5:5494 doi: 10.1038/ ncomms6494 (2014). 